\title{
Improved Design of a DFB Raman Fibre Laser
}

\author{
Youfang $\mathrm{Hu}^{1}$, Neil G R Broderick ${ }^{2}$ \\ Optoelectronic Research Centre, University of Southampton, Highfield, Southampton, \\ SO17 1BJ, United Kingdom
}

1. Corresponding author

Postal address:

Optoelectronic Research Centre, University of Southampton, Highfield, Southampton, SO17 1BJ, United Kingdom

E-mail: yfh@orc.soton.ac.uk

Tel: $+44(0) 2380592610$

Fax: +44 (0)238059 3142

2. E-mail: ngb@orc.soton.ac.uk

\begin{abstract}
:
A Raman fibre laser based on phase shifted DFB structures is modelled for the first time. Using parameters of realistic devices, the model predicts low-threshold and highly-efficient laser output. The change of position and width of the phase shift were found to have a substantial impact on laser performance.
\end{abstract}

\section{Key words:}

Distributed feedback, Fibre Bragg grating, Highly nonlinear fibre, Raman fibre laser, Phase shift 


\section{Introduction}

Nonlinear effects in Bragg gratings have been the extensively studied both theoretically and experimentally since Winful et. al. [1] predicted that such devices are bistable. Most of this work has concentrated on self-phase modulation effects (i.e. single wavelengths interactions) with only a few studies looking at multiple wavelength interactions (see for example [2, 3]) and the Kerr nonlinearity. However in fibres other nonlinearities are present leading to novel interactions in fibre Bragg gratings (FBG). The Raman effect can be used to create a Raman Gap Soliton [4] and even can be used to make a Raman pumped fibre DFB laser [5].

Raman fibre lasers are particularly attractive since they provide a source of coherent high intensity light at wavelengths where no conventional lasing material works. However typically Raman fibre lasers have a long cavity length ( $\mathrm{kms}$ of fibre) making them extremely sensitive to environmental fluctuations and so shorter devices are desirable. In this context the Raman DFB laser seems particularly attractive since a linear analysis of such structures shows that the laser's threshold is inversely proportional to the cube of grating length, $L^{3}$, and the square of grating coupling coefficient, $k^{2}$ [5]. However in order to achieve a practical threshold $(<1 \mathrm{~W})$, long $(L>1 \mathrm{~m})$ and strong gratings are required, which makes experimental observation of these lasers extremely difficult. Also, the two modes with the lowest gain threshold will compete when threshold is reached, which may destabilise the laser's output. Finally, the lasing frequency is expected to be linearly dependant on the pump power, hence, a slight power fluctuation in the cavity will detune the output signal from resonance and the output power will decay. Therefore, a more practical laser design is highly desirable.

In conventional DFB lasers these problems have been studied extensively, and a common solution is the introduction of a $\pi$-shift in the spatial phase of the grating. Low threshold, single mode lasing in $\pi$-shifted DFB structure was first studied both experimentally and theoretically in semiconductors [6] and then in fibres (these models also considered the fibre Kerr nonlinearity $[7,8])$. However, this kind of structure has not been introduced into the design of Raman fibre lasers. In our study, we demonstrate, for the first time, an optimised design of $\pi$ - 
shift structure in a DFB Raman fibre laser, which offers substantial benefit compared to a uniform DFB structure.

\section{Theoretical model}

The Raman DFB laser we consider here is formed by a long fibre Bragg grating. The laser is optically pumped from left hand side $(z=0)$ of the grating at a wavelength, $\lambda_{p}$. As the pump wave propagates through the fibre, the Raman process transfers energy from the pump wave to Stokes waves, $\lambda_{s}$, which is close to the grating's Bragg wavelength, $\lambda_{B}$. As a result, the Stokes waves propagates in both the forward and backward directions while the pump wave passes through the fibre without interacting with the grating since it is very far from the Bragg resonance. The slow varying amplitudes of the pump $\left(A_{p}\right)$, forward Stokes $\left(A_{f}\right)$, and backward Stokes $\left(A_{b}\right)$ waves inside the laser are governed by the following set of nonlinear coupled wave equations [5]:

$$
\begin{aligned}
& \frac{\partial A_{p}}{\partial z}+\frac{1}{v_{p}} \frac{\partial A_{p}}{\partial t}=-\frac{g_{p}}{2}\left(\left|A_{f}\right|^{2}+\left|A_{b}\right|^{2}\right) A_{p}+i \gamma_{p}\left(\left|A_{p}\right|^{2}+2\left|A_{f}\right|^{2}+2\left|A_{b}\right|^{2}\right) A_{p}-\frac{\alpha_{l p}}{2} A_{p} \\
& \frac{\partial A_{f}}{\partial z}+\frac{1}{v_{s}} \frac{\partial A_{f}}{\partial t}=\frac{g_{s}}{2}\left|A_{p}\right|^{2} A_{f}+i \gamma_{s}\left(2\left|A_{p}\right|^{2}+\left|A_{f}\right|^{2}+2\left|A_{b}\right|^{2}\right) A_{f}+i \kappa A_{b}-\frac{\alpha_{l s}}{2} A_{f} \\
& -\frac{\partial A_{b}}{\partial z}+\frac{1}{v_{s}} \frac{\partial A_{b}}{\partial t}=\frac{g_{s}}{2}\left|A_{p}\right|^{2} A_{b}+i \gamma_{s}\left(2\left|A_{p}\right|^{2}+2\left|A_{f}\right|^{2}+\left|A_{b}\right|^{2}\right) A_{b}+i \kappa^{*} A_{f}-\frac{\alpha_{l s}}{2} A_{b}
\end{aligned}
$$

where, $v_{g}$ and $v_{s}$ are the group velocities of pump and Stokes waves. For a short device $(<1 \mathrm{~m})$, the material and waveguide dispersion and walk-off effect of the three waves are ignorable; hence, the $v_{g}$ and $v_{s}$ are considered to be equal; $g_{s}$ is the Raman gain coefficient for Stokes waves and the gain depletion coefficient for pump wave is given by $g_{p}=g_{s}\left(\lambda_{s} / \lambda_{p}\right)$; the nonlinear coefficients are defined by $\gamma_{p}=2 \pi n_{2} / \lambda_{p}$ and $\gamma_{s}=2 \pi n_{2} / \lambda_{s} ; \kappa$ is the grating's coupling constant; $\alpha_{l p}$ and $\alpha_{l s}$ are the linear losses of pump and Stokes waves. The fields are normalised so that $I_{p, f, b}=\left|A_{p, f, b}\right|^{2}=P_{p, t, b} / A_{\text {eff, }}$ where, $A_{\text {eff }}$ is the effective mode area for the fibre.

Early theoretical work shows that an abrupt $\pi$ phase change of the coupling coefficient, $\kappa$, inside the DFB laser enables single-mode low-threshold lasing [2-4] by creating a defect state inside the grating's bandgap at a frequency determined by the phase shift position $z_{\pi}$. More 
realistically, there must be a small physical width $d z$ over which this phase change occurs. Since the width and position of the phase shift substantially influences the laser's performance [7-10] it is crucial to be able to optimise these parameters. Thus in our study we modelled the phase shift as:

$$
\kappa= \begin{cases}\kappa_{0} & \left(0 \leq z \leq z_{\pi}-d z / 2\right) \\ \kappa_{0} \exp \left(i \pi\left[\left(z-z_{\pi}\right) / d z+1 / 2\right]\right) & \left(z_{\pi}-d z / 2<z<z_{\pi}+d z / 2\right) \\ -\kappa_{0} & \left(z_{\pi}+d z / 2<z<L\right)\end{cases}
$$

where $\kappa_{0}$ is the grating's strength which is proportional to the refractive index modulation; $d z$ is the phase shift width; and $z_{\pi}$ is the distance from the grating's left hand side $(z=0)$ to the phase shift position.

To solve equations (1)-(3), we used a fourth-order implicit Runge-Kutta algorithm as proposed in [11]. As we were interested in the $\mathrm{CW}$ response of the grating we took $A_{p}(0, t)=A_{0}$ where $A_{0}$ is the constant Raman pump power and solved the coupled mode equations for both the output power and the mode profile. We kept the full time-dependence in the equations since we expected the nonlinearity to cause the output to become unstable above some threshold power and also to be able to model the transient behaviour of the laser as it approached threshold.

In our simulation, we used the following realistic values for the parameters. Firstly, taking the values for silica gives a Raman gain coefficient of $g_{R}=0.7 \mathrm{e}-13 \mathrm{~m} / \mathrm{W}$ and a nonlinear refractive index of $n_{2}=3.2 \mathrm{e}-20 \mathrm{~m}^{2} \mathrm{~W}$. The pump wavelength is $\lambda_{p}=1550 \mathrm{~nm}$ corresponding to the peak gain of EDFAs resulting in a Stokes wavelength $\lambda_{s}=1662 \mathrm{~nm}$. In order to minimise the lasing threshold, we set $A_{\text {eff }}=2 \mu \mathrm{m}^{2}$, such a small fibre mode size is achievable using microstructured fibres with a Ge-doped core as demonstrated by [12]. Compared with previous work [5], we examined relatively short strong gratings with a length of $20 \mathrm{~cm}$ and a strength $\kappa_{0}=60 \mathrm{~m}^{-1}$. Using short gratings ensures that the grating's uniformity is easily maintained along the entire length while the value of $\kappa$ is a compromise between what can easily be fabricated and having to ensure a low threshold. Finally the linear losses for both the pump and Stokes waves in 
such a small core fibre are considered to be higher than for a standard fibre and we took $\alpha_{1 p}=\alpha_{1 s}=0.2 \mathrm{~dB} / \mathrm{m}$.

\section{Modelling results and discussion}

The typical behaviour of the laser is shown in Fig. 1 , where $z_{\pi}=11.8 \mathrm{~cm}, d z / L=0$ and $P_{\text {pump }}=\left|A_{p}\right|^{2} A_{\text {eff }}=3 \mathrm{~W}$. The laser's output initially builds up exponentially, but as the laser approaches a steady state, the output signal builds up faster and faster until it suddenly saturates. Due to the offset of $z_{\pi}$ from the grating centre, the Stokes wave output from right hand side of the laser, $P_{r h s}$, exceeds that from left hand side, $P_{l h s}$, by more than one order of magnitude. As expected due to the phase shift this laser exhibited a non-uniform cavity power distribution in steady state (Fig. 1b), which is contrary to Perlin's prediction [5] that a flat cavity field distribution is the only field configuration for lasing in a nonlinear DFB laser. The laser cavity power distribution reaches its maximum power $P_{\pi}$ at the $\pi$-shift position and the power decays exponentially towards both cavity edges, which is typical for a $\pi$-shift DFB laser.

For a better understanding of the laser's behaviour in steady state, we examined the cavity power distribution of the Stokes waves for different widths, $d z$, of the phase shift, as shown in Fig. 2. For comparison we also show the linear case $\left(n_{2}=0\right)$ with $d z=0$, corresponding to a conventional DFB laser. Here, we can see that the nonlinearity plays a significant role in shaping the power distribution. Comparing the linear and nonlinear cases when $d z=0$, we see that the nonlinearity flattens the power distribution and reduces the peak cavity power, $P_{\pi}$, by a factor of $\sim 5$ but increases the power at $z=L, P_{r h s}$, by $60 \%$. As the phase shift region becomes wider (increase of $d z / L$ from 0 to 0.16 ), the cavity power's peak still stays at the $\pi$ shift position, while the cavity power distribution becomes even flatter with $P_{\pi}$ dropping by $20 \%$ and $P_{r h s}$ increasing by $80 \%$. Evidently, a $\pi$-shift Raman DFB fibre laser operates more efficiently with a flatter cavity power distribution. However, the power distribution need not to be strictly flat over the major part of the cavity, as described in [5], to enable lasing.

Next, we investigated the laser's output characteristics by varying the pump power for different phase shift widths and these results are shown in Fig. 3. Fig. 3 shows that the laser's 
threshold can be reduced below $1 \mathrm{~W}$ by increasing $d z$ making it a practical device. In comparison, a grating with the same parameters but without the phase shift has a threshold of $>20 \mathrm{~W}$. It is also evident from Fig. 3 that the increase of the width of the phase shift, $d z / L$, from 0 to 0.16 corresponds to an increase of laser's slope efficiency from $50 \%$ to $82 \%$ and a decrease of lasing threshold. For our choice of parameters stable lasing occurs for pumping levels below about $4 \mathrm{~W}$ which is the cut-off in the graph. Increasing the pump power still further results in nonlinear instability (self-pulsation and chaos) in the output. Similarly, as the phase shift region becomes even wider $(d z / L>0.16)$, laser does not reach a steady state, rather, it becomes unstable above threshold.

In order to optimise our laser, we investigated its performance for different positions of the phase shift. Our model showed steady state output for $10.6 \mathrm{~cm}<z_{\pi}<12.6 \mathrm{~cm}$, outside of which the laser output was unstable. Fig. 4 shows the right hand side output of the laser within the stable region for $z_{\pi}$ when the pumping power was fixed at $3 \mathrm{~W}$. It can be seen that the change of the output, $P_{r h s}$, with respect to $z_{\pi}$ is very small, particularly for small $d z$ (diamonds), as is expected for a phase shifted DFB laser with a large value of $\kappa L$. In our case, $\kappa L=12$, corresponding to a short effective cavity region $\left(L_{\text {eff }} / L=0.08\right)$ centred at $z_{\pi}$ according to [9]. Hence, the effective reflectivities of both ends of the laser are not much affected when this short effective grating region is slightly shifted around $z_{\pi}$. As the output efficiency is directly related to the reflectivity of laser cavity, the former is also not much affected. In comparison, the laser's output was much more sensitive to the change of phase shift width, $d z$. This is due to the fact that most of the cavity's energy is distributed within and around the phase shift region and thus increasing the width of this region has a substantial impact on the laser's output.

Finally, we estimated the lasing thresholds for different values of $z_{\pi}$ and the results are shown in Fig. 5. Fig. 5 shows that the threshold increases continuously as phase shift position, $z_{\pi}$, shifts away from grating centre $(z=10 \mathrm{~cm})$. This can be understood from the fact that the round trip reflectivity, $R_{l h s} \times R_{r h s}$, has a maximum value, i.e. lowest reflection loss, when $z_{\pi}=L / 2$ and monotonically decreases as $z_{\pi}$ shifts from the grating's centre. Thus as the cavity losses 
increase, the laser threshold also increases. The lasing threshold is also affected by the value of $d z$. As the phase shift region becomes wider, the region of high gain is increased since the effective cavity region is stretched. Thus we see that, for a fixed $z_{\pi}$, the threshold decreases for increasing $d z$. However there is a drawback to increasing $d z$ since for a fixed length laser, increasing $d z / L$ means decreasing the grating length on both sides of the phase shift, which results in increased losses on both ends of the laser. Therefore, the rate of decrease of lasing threshold is reduced as $d z / L$ becomes larger. This trade-off effect can be seen in Fig. 5 , for $z_{\pi}$ $>11.6 \mathrm{~cm}$, where the slope of the curves increase. It should be noted that although the particular optimum values for $d z$ and $z_{\pi}$ will depend on the precise grating parameters the trends outlined here are universally valid and can be used as a design tool for making improved DFB Raman lasers.

\section{Conclusion}

In conclusion, we have demonstrated a practical design of a Raman DFB fibre laser based on a relatively short $(<20 \mathrm{~cm}), \pi$-shift fibre Bragg grating. The laser showed highly efficient steady-state output from one end the laser cavity when the grating's phase shift position was slightly offset from the grating centre. The laser's cavity distribution was flattened and the output was increased as a result of high nonlinearity of the fibre. By optimising the grating's phase shift width and position, the laser's threshold can be lower than $1 \mathrm{~W}$ and its slope efficiency higher than $80 \%$. We have identified clear design rules for optimising the position and width of the phase shift. Finally since all the parameters used in the model have realistic values that are readily achievable we hope that a real device will be presented in the near future. 


\section{References:}

[1] H. G. Winful, J. H. Marburger, E. Garmire, Appl. Phys. Lett. 35 (1979) 379-381.

[2] C. M. de Sterke, Opt. Lett. 17 (1992) 914-916.

[3] N. G. R. Broderick, D. Taverner, D. J. Richardson, M. Ibsen, R. I. Laming, Phys. Rev. Lett. 79 (1997) 4566-4569.

[4] H. G. Winful, V. E. Perlin, Phys. Rev. Lett. 84 (2000) 3586-3589.

[5] V. E. Perlin, H. G. Winful, IEEE J. Quantum Electron. 37 (2001) 38-47.

[6] J. Carroll, J. Whiteaway, D. Plumb, Distributed Feedback Semiconductor lasers, The Institute of Electrical Engineers, Herts, 1998.

[7] V. C. Lauridsen, J. H. Povlsen, P. Varming, Electron. Lett. 34 (1998) 2028-2030.

[8] V. C. Lauridsen, J. H. Povlsen, P. Varming, Electron. Lett. 35 (1999) 300-302.

[9] C. M. de Sterke, K. R. Jackson, B. D. Robert, J. Opt. Soc. Am. B, 8 (1991) 403412.

[10]K. Yelen, L. M. B. Hickey, M. N. Zervas, IEEE J. Quantum Electron. 40 (2004) 711-720.

[11]K. Yelen, M. N. Zervas, L. M. B. Hickey, J. Lightwave Technol. 23 (2005) 32-43.

[12] M. C. P. Huy, G. Laffont, V. Dewynter, P. Ferdinand, P. Roy, J. L. Auguste, D. Pagnoux, W. Blanc, B. Dussardier, Opt. Lett. 32 (2007) 2390-2392. 
Figure 1:

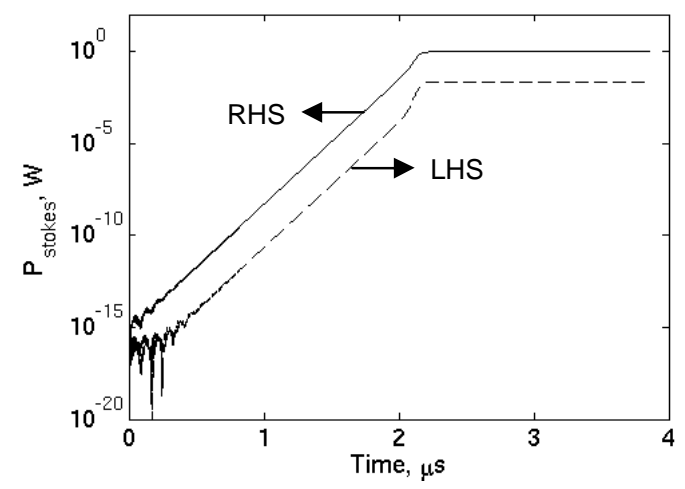

(a)

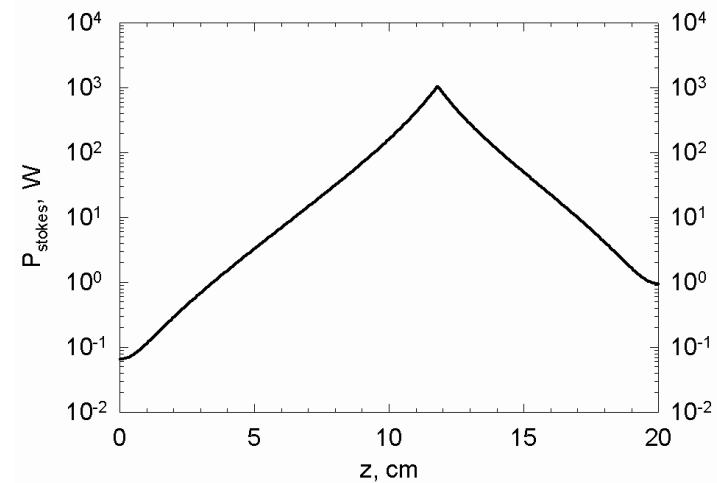

(b)

Fig. 1 (a) Stokes wave output power versus time and (b) Cavity power distribution (the sum of forward wave and backward Stokes wave) at steady state, $z_{\pi}=11.8 \mathrm{~cm}, d z / L=0, P_{\text {pump }}=3 \mathrm{~W}$. 


\section{Figure 2:}

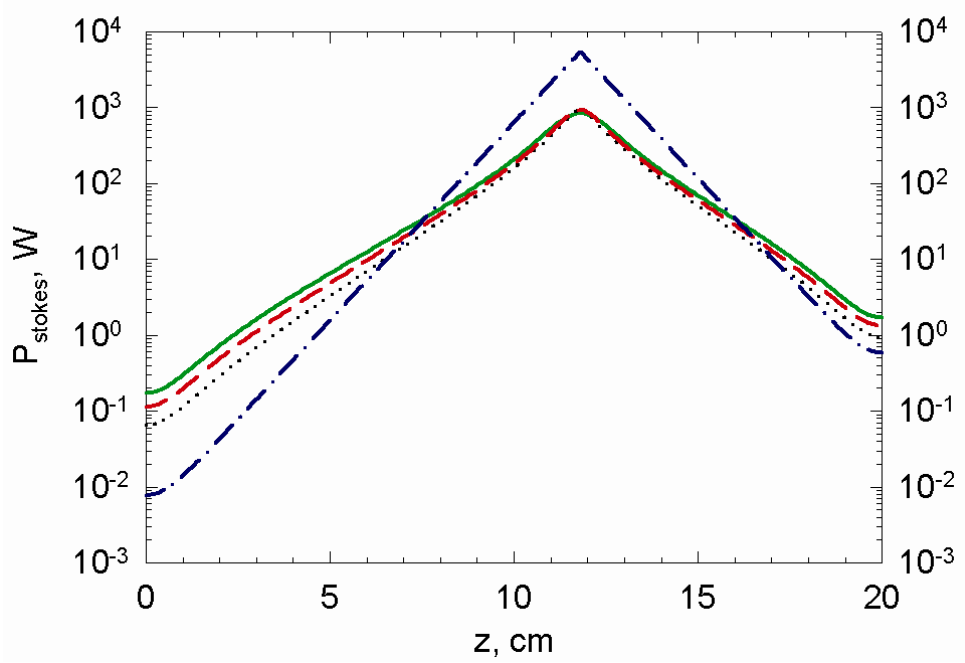

Fig. 2 Stokes wave power distribution (the sum of forward wave and backward wave) inside the cavity in steady state, $z_{\pi}=11.8 \mathrm{~cm}, P_{\text {pump }}=3 W$. Solid line: $d z / L=0.16$. Dashed line: $d z / L=0.08$. Dotted line: $d z / L=0$. Dash dotted line: $d z / L=0, n_{2}=0$. 
Figure 3:

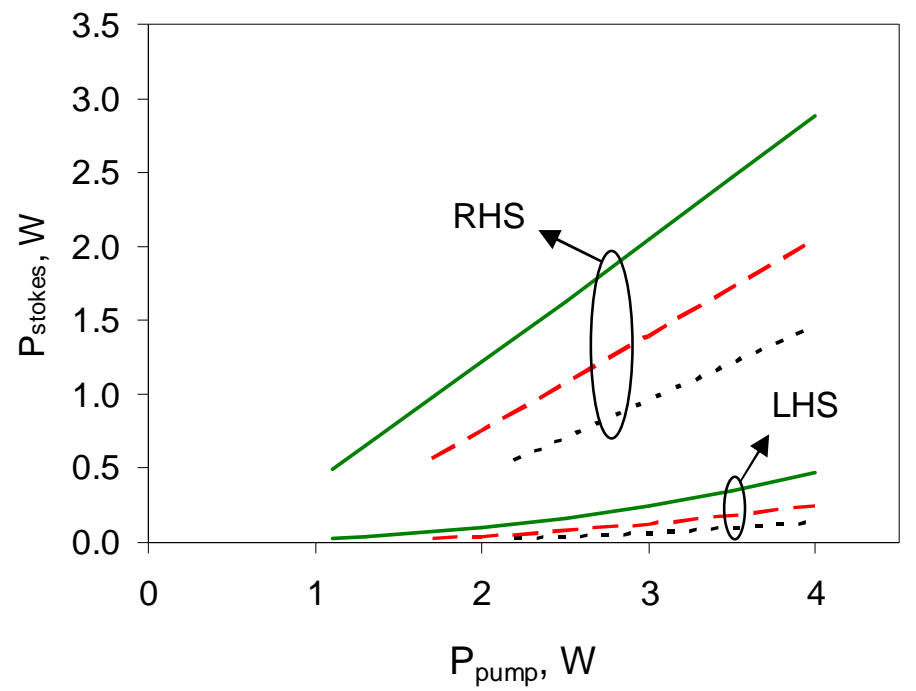

Fig. 3 Stokes output power versus CW pump power, $z_{\pi}=11.8 \mathrm{~cm}$; solid line: $d z / L=0.16$, long dashed line: $d z / L=0.08$, short dashed line: $d z / L=0$. 
Figure 4:

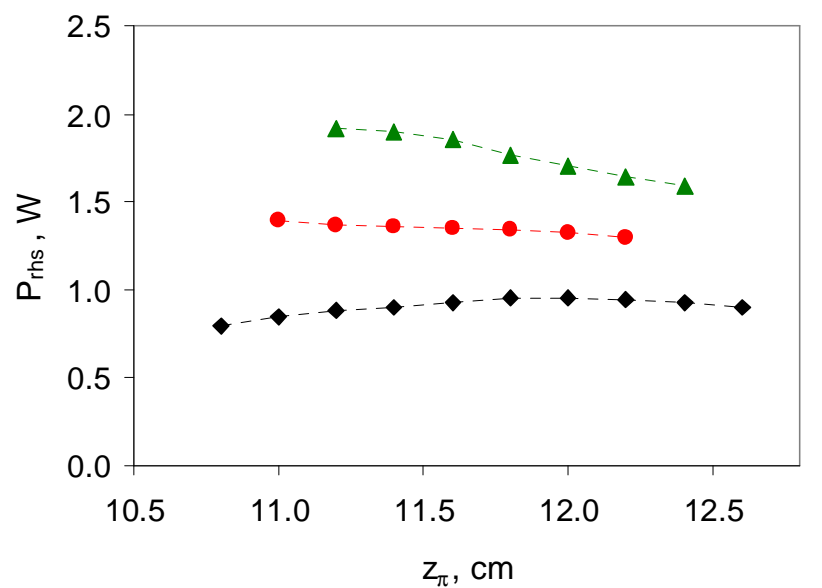

Fig. 4 Stokes wave output from right hand side of the laser, $P_{r h s}$, versus phase shift position, $z_{\pi}$, within stable output region $\left(P_{\text {pump }}=3 W\right)$. Triangle: $d z / L=0.16$. Circle: $d z / L=0.08$. Diamond: $d z / L=0$. 
Figure 5:

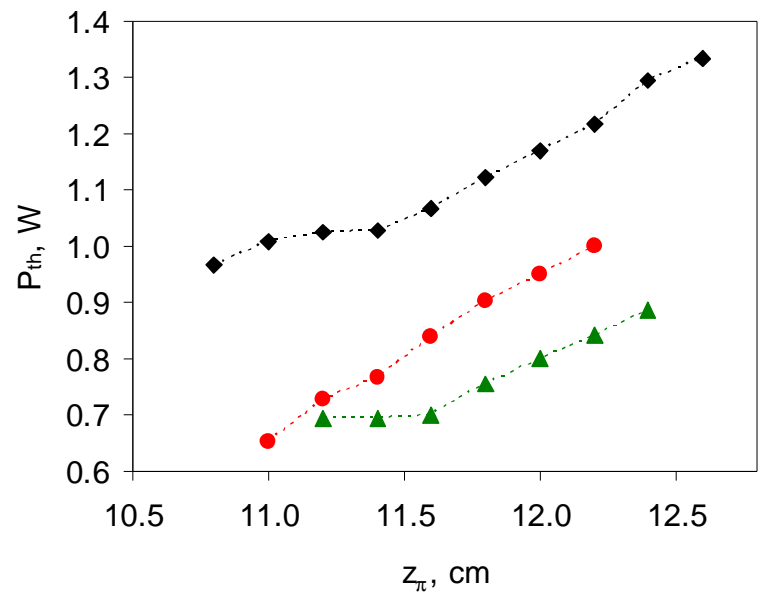

Fig. 5 Laser threshold, $P_{t h}$, versus phase shift position, $z_{\pi}$, within the stable output region. Triangle: $d z / L=0.16$. Circle: $d z / L=0.08$. Diamond: $d z / L=0$. 